\title{
Overexpression of dilp2 causes nutrient-dependent semi-lethality in Drosophila
}

\author{
Yukiko Sato-Miyata ${ }^{1+}$, Keigo Muramatsu ${ }^{1+}$, Masabumi Funakoshi ${ }^{1}$, Manabu Tsuda $^{2}$ and \\ Toshiro Aigaki ${ }^{*}$ \\ ${ }^{1}$ Cellular Genetics Laboratory, Department of Biological Sciences, Tokyo Metropolitan University, Tokyo, Japan \\ ${ }^{2}$ Department of Liberal Arts and Human Development, Faculty of Health and Social Services, Kanagawa University of Human Services, Yokosuka, Japan
}

Edited by:

Xanthe Vafopoulou, York University,

Canada

Reviewed by:

Christen Kerry Mirth, Fundação

Calouste Gulbenkian, Portugal

Yih-woei Fridell, University of

Connecticut, USA

*Correspondence:

Toshiro Aigaki, Department of Biological Sciences, Tokyo

Metropolitan University, 1-1

Minami-osawa, Hachioji, Tokyo

192-0397, Japan

e-mail: aigaki-toshiro@tmu.ac.jp

${ }^{\dagger}$ These authors have contributed equally to this work.
Insulin/insulin-like growth factor (IGF) plays an important role as a systemic regulator of metabolism in multicellular organisms. Hyperinsulinemia, a high level of blood insulin, is often associated with impaired physiological conditions such as hypoglycemia, insulin resistance, and diabetes. However, due to the complex pathophysiology of hyperinsulinemia, the causative role of excess insulin/IGF signaling has remained elusive. To investigate the biological effects of a high level of insulin in metabolic homeostasis and physiology, we generated flies overexpressing Drosophila insulin-like peptide 2 (Dilp2), which has the highest potential of promoting tissue growth among the $/ / p$ genes in Drosophila. In this model, a UAS-Dilp2 transgene was overexpressed under control of sd-Gal4 that drives expression predominantly in developing imaginal wing discs. Overexpression of Dilp2 caused semi-lethality, which was partially suppressed by mutations in the insulin receptor $(\operatorname{In} R)$ or $A k t 1$, suggesting that dilp2-induced semi-lethality is mediated by the PI3K/Akt1 signaling. We found that dilp2-overexpressing flies exhibited intensive autophagy in fat body cells. Interestingly, the dilp2-induced autophagy as well as the semi-lethality was partially rescued by increasing the protein content relative to glucose in the media. Our results suggest that excess insulin/IGF signaling impairs the physiology of animals, which can be ameliorated by controlling the nutritional balance between proteins and carbohydrates, at least in flies.

Keywords: Drosophila insulin-like peptides, insulin-like growth factor signaling, hyperinsulinemia, growth regulation, autophagy, protein-to-carbohydrate ratio

\section{INTRODUCTION}

In mammals, the peptide hormone insulin promotes glucose uptake in muscles and adipose tissues, induces cell growth and proliferation, and stimulates glyconeogenesis, lipogenesis, and protein synthesis (Saltiel and Kahn, 2001). The insulin/insulinlike growth factor (IGF) signal is evolutionally conserved throughout multicelluar organisms (Skorokhod et al., 1999). In insects, Drosophila has been extensively used as a model system to study insulin signaling, which plays an important role in regulating organ growth and the final size of the organism.

Drosophila possesses eight insulin-like peptides (Dilps), which can activate the Drosophila insulin receptor, InR (Brogiolo et al., 2001). Among the Drosophila insulin-like peptides (Ilps), dilp2 is the most highly expressed and it has the highest potential for promoting tissue growth (Ikeya et al., 2002; Rulifson et al., 2002; Broughton et al., 2005). It has been demonstrated that reduction of dilp2 increases the content of the insect blood sugar, trehalose, in adult flies, suggesting that dilp2 regulates glucose homeostasis in Drosophila as it also does in mammals (Broughton et al., 2008). Furthermore, reduction of dilp2 expression has been shown to increase lifespan, indicating that dilp2 plays an important role in lifespan determination (Broughton et al., 2008).

On the other hand, excess activation of insulin signaling could impair the physiology of organisms. In humans, it has been proposed that increased levels of insulin in the blood is a primary cause of Type 2 diabetes associated with hypertension and cancers (Novosyadlyy and LeRoith, 2010). In fact, hyperinsulinemia, which is an excessive level of insulin in the blood, is often seen in several metabolic diseases, such as Type 2 diabetes mellitus (Samuel and Shulman, 2012). However, the coexistence of hyperglycemia, insulin resistance, and other hormonal and metabolic changes in patients with Type 2 diabetes makes it difficult to understand the causative role of excess insulin signaling in the pathophysiology of hyperinsulinemia (Corkey, 2012). Several animal models for hyperinsulinemia have been developed by overexpressing InR or IGFR in some tissues, by the short-time administration of insulin, or by feeding animals a high-sugar diet (Musselman et al., 2011). Although these models have contributed to elucidating the molecular mechanisms that regulate insulin/IGF signaling, how hyperinsulinemia affects animal physiology has remained elusive.

It has been demonstrated that dietary composition also affects physiology and lifespan of individuals. In Drosophila, the balance of protein to carbohydrate intake is one of the critical determinants for lifespan and fecundity (Lee et al., 2008; Skorupa et al., 2008; Lushchak et al., 2012). For example, flies maintained with glucose-rich/protein-poor food generally become obese with age and exhibited a shorten lifespan and vise versa. Although 
insulin/IGF signaling plays crucial roles in regulation of glucose uptake, how the signal influences the dietary compositiondependent physiological changes is unclear.

To investigate how excess insulin affects insect physiology, we generated transgenic flies with a high level of dilp2 and analyzed their phenotypes. Overexpression of dilp2 increased the body size and caused semi-lethality. These phenotypes were partially suppressed by mutations in the insulin/IGF signaling pathway components, thereby suggesting that hyperactivation of the insulin/IGF signaling is toxic to flies. We found that dilp2-overexpressing flies exhibited intensive autophagy in fat body cells. Interestingly, increasing the protein content relative to glucose in the media partially rescued the dilp2-induced semi-lethality and autophagy. Our results suggest that excess insulin/IGF signaling impairs the physiology of animals, but it can be ameliorated by controlling the nutritional balance between proteins and carbohydrates, at least in flies.

\section{MATERIALS AND METHODS FLY STOCKS AND MEDIA}

UAS-dilp2 (Brogiolo et al., 2001), $A k t 1^{1}$ (Stocker et al., 2002), and $I n R^{304}$ (Brogiolo et al., 2001) were kindly provided by Dr. E. Hafen. PTEN ${ }^{d j 189}$ was a gift from Dr. D. Pan (Gao et al., 2000). Tor K17004 (Oldham et al., 2000), S6K 07064 (Montagne et al., 1999), and $M\{3 x P 3-R F P . a t t P\} Z H-51 D$ and $M\{3 x P 3-R F P . a t t P\} Z H-68 E$ (Bischof et al., 2007) were obtained from the Bloomington Stock Center. Flies were reared at $25^{\circ} \mathrm{C}$ on a standard cornmeal medium [3.6\% neutralized yeast (Asahi Breweries, LTD. Y-4), $8.1 \%$ cornmeal, $10 \%$ glucose, and $0.7 \%$ agar] with propionic acid and $n$-butyl $p$-hydroxybenzoate as mold inhibitors, unless otherwise stated. We used different medium for the convenience of preparation. Drosophila Instant Medium (Formula 4-24, Carolina Biological. Supply, Burlington, NC) was used to as a basal medium to prepare media containing different concentration of yeast extracts: $2 \mathrm{~g}$ of Drosophila Instant Medium was mixed with $5 \mathrm{ml}$ of Bacto ${ }^{\mathrm{TM}}$ Yeast Extract (Difco Laboratories, Detroit, MI, USA) dissolved in water at four different concentrations $(0,10,20$, and $40 \mathrm{~g} / \mathrm{L})$. Standard cornmeal agar medium was used to prepare media containing glucose at four different concentrations $(0,100,200$, and $300 \mathrm{~g} / \mathrm{L})$.

\section{GENETIC INTERACTION EXPERIMENTS}

To facilitate genetic interaction experiments, we generated a stock, sd-Gal4/sd-Gal4; UAS-dilp2/TM6B, tub-Gal80, in which GAL4dependent expression of dilp2 is repressed by GAL80. The stock is convenient to test the effects of mutations on the phenotype caused by overexpression of dilp2. To make an internal control, we made flies heterozygous for a mutation with a homologous chromosome marked with RFP: second chromosome-linked mutations (Tor ${ }^{K 17004}$ and $P T E N^{d j 189}$ ) and third chromosome-linked mutations $\left(A k t 1^{1}, I n R^{304}\right.$, and $\left.S 6 K^{07064}\right)$ were crossed to $M\{3 x P 3-$ RFP.attP $\} Z H-51 D$ and $M\{3 x P 3-R F P . a t t P\} Z H-68 E$, respectively. The F1 progenies (mutations/3xP3-RFP) were crossed to $s d$ Gal4/sd-Gal4; UAS-dilp2/ TM6B, tub-Gal80 flies. Numbers of resulting progenies ( $s d-G a l 4 /+$; UAS-dilp2/+ with mutations) and their sibling controls (sd-Gal4/+; UAS-dilp2/+ with $3 \times P 3-$ $R F P)$ were counted and calculated relative viabilities.

\section{MEASUREMENT OF BODY WEIGHT AND WING SIZE}

The adult flies were weighed using an Analytical Semi-Micro Balance (A\&D Company, Tokyo, Japan). To measure wing size, the right wings of the adult flies were torn off by using forceps and mounted onto a microscopic slide using a drop of Fly Line Dressing (TIEMCO, Tokyo, Japan), a silicone grease with very low surface tension (Tsuda et al., 2010). The wings were photographed using a MZ APO stereomicroscope (Leica, Wetzlar, Germany) equipped with a DP50 digital camera (Olympus, Tokyo, Japan) at a constant magnification. The areas of the wings were measured by using ImageJ software (NIH).

\section{QUANTITATIVE REAL-TIME PCR}

Total RNA from the adult flies was extracted using TRIzol ${ }^{\circledR}$ (Qiagen, Valencia, CA, USA) and it was reverse-transcribed using ReverTra Ace $^{\circledR}$ (Toyobo, Osaka, Japan). Quantitative-PCR reactions were carried out using SYBR ${ }^{\circledR}$ Premix Ex Taq ${ }^{\mathrm{TM}}$ (Takara Bio, Otsu, Japan).

\section{WESTERN BLOT ANALYSIS}

The adult flies were homogenized in SDS sample buffer $(12.5 \mathrm{mM}$ Tris ( $\mathrm{pH} 6.8$ ), 20\% glycerol, 4\% SDS, 2\% 2-mercaptoethanol, and $0.001 \%$ bromophenol blue) and boiled for $10 \mathrm{~min}$ at $95^{\circ} \mathrm{C}$. The samples were separated by $10 \%$ SDS-PAGE and transferred to PVDF membranes (GE Healthcare, Buckinghamshire, UK). After blocking with 5\% bovine serum albumin (Sigma-Aldrich, St. Louis, MO, USA), the membranes were incubated with a primary antibody in Tris-buffered saline (TBS) containing Tween-20 (TBST) overnight at $4^{\circ} \mathrm{C}$ and then with a secondary antibody in TBST for $1 \mathrm{~h}$ at $25^{\circ} \mathrm{C}$. The signals were detected with an ECL-plus kit (GE Healthcare). As primary antibodies, rabbit anti-phospho-Akt antibody (Cell Signaling Technology, Danvers, MA, USA), rabbit anti-phospho-p70 S6 kinase (Cell Signaling Technology), and mouse anti- $\alpha$-tubulin (Sigma-Aldrich) were used at dilutions of 1:1000, 1:1000, and 1:5000, respectively. HRP-conjugated anti-rabbit IgG (Cell Signaling Technology) and HRP-conjugated anti-mouse IgG (GE Healthcare) were used as secondary antibodies at dilutions of 1:2000 and 1:1000, respectively.

\section{LIQUID CHROMATOGRAPHY COUPLED TO TANDEM MASS SPECTROMETRY (LC-MS/MS)}

LC-MS/MS was used to determine the concentrations of glucose, trehalose, glucose metabolites, and free amino acids. Ten female flies were weighted and homogenized with $75 \%$ acetonitrile on ice. The homogenates were centrifuged for $10 \mathrm{~min}$ at $2400 \times \mathrm{g}$ and the supernatant was re-centrifuged for $10 \mathrm{~min}$ at $1200 \times \mathrm{g}$. The supernatant was evaporated and dissolved to mobile phase (10 mM DBAA, Tokyo Chemical Industry, Tokyo, Japan), in $\mathrm{H}_{2} \mathrm{O}$ $(\mathrm{pH}$ 4.75). After centrifuging at $1200 \times \mathrm{g}$ to remove residue, the supernatants were collected and used for metabolome analysis with a Waters LC-QTofMS system composed of LC (Acquity UPLC) and MS (Xevo ${ }^{\mathrm{TM}}$ QTofMS) (Waters, Milford, MA, USA). The metabolites were separated on an Acquity ${ }^{\circledR}$ UPLC HSS T3 column $(2.1 \AA \sim 100 \mathrm{~mm}, 1.8 \mu \mathrm{m}$; Waters). For the carbon metabolites, the columns were equilibrated with $10 \mathrm{mM}$ 
DBAA (Tokyo Chemical Industry) in $\mathrm{H}_{2} \mathrm{O}$ ( $\mathrm{pH}$ 4.75), and the compounds were eluted with an increasing gradient of acetonitrile. The total run time was $20 \mathrm{~min}$. The MS system was equipped with a dual electrospray ionization probe and operated in the negative ion mode with the source temperature at $120^{\circ} \mathrm{C}$. For the amino acids, the analytes were separated by a gradient of mobile phase ranging from water containing $0.05 \%$ acetic acid to methanol over a $15 \mathrm{~min}$ run. The capillary voltage and the cone voltage for electrospray ionization was maintained at $0.7 \mathrm{kV}$ and $15 \mathrm{~V}$ for negative mode detection and at $0.7 \mathrm{kV}$ and $13 \mathrm{~V}$ for positive mode detection, respectively. The source temperature and the desolvation temperature were set at 120 and $350^{\circ} \mathrm{C}$, respectively. Nitrogen was used as both the cone gas $(50 \mathrm{l} / \mathrm{h})$ and the desolvation gas $(600 \mathrm{l} / \mathrm{h})$ and argon was used as the collision gas. For accurate mass measurement, the mass spectrometer was calibrated with sodium formate solution (range $\mathrm{m} / \mathrm{z} 50-1000$ ) and monitored by the intermittent injection of the lock mass leucine enkephalin $([\mathrm{M}+$ $\mathrm{H}]+=556.2771 \mathrm{~m} / \mathrm{z}$ and $[\mathrm{M}-\mathrm{H}]-=554.2615 \mathrm{~m} / \mathrm{z})$ in real time. The MS data were analyzed using QuanLynx ${ }^{\mathrm{TM}}$ (Waters). The compounds were identified based on their retention time, their $\mathrm{m} / \mathrm{z}$ ratio, and the MS/MS spectrum of standard reference materials.

\section{MEASUREMENT OF PROTEIN CONTENT}

The concentration of soluble protein was measured using the Bio-Rad protein assay reagent. At least three trials were carried out for each genotype.

\section{TRIGLYCERIDE MEASUREMENT}

Ten adult flies were weighed and homogenized in $1 \%$ Triton-X. The homogenates were heated for $10 \mathrm{~min}$ at $70^{\circ} \mathrm{C}$ and stored at $-80^{\circ} \mathrm{C}$. After thawing on ice, the samples were centrifuged for $10 \mathrm{~min}$ at $14,700 \times \mathrm{g}$ at $25^{\circ} \mathrm{C}$. The amount of triglycerides (TAG) was determined using a Serum Triglyceride Determination Kit (TR0100; Sigma-Aldrich, St. Louis, MO, USA). All data were normalized with soluble protein contents in homogenates. At least three samples were used to determine the average amount of triglycerides.

\section{AUTOPHAGY STAINING}

The fat bodies were dissected from the early third larvae in PBS and incubated with LysoTracker ${ }^{\circledR}$ Red DND-99 (Molecular Probes $^{\circledR}$, Life Technologies, Grand Island, NY, USA) at a 1:1000 dilution in PBS for $1 \mathrm{~min}$ at room temperature. After a brief wash in PBS, the sample was observed under a Nikon C1 laser scanning confocal microscope.

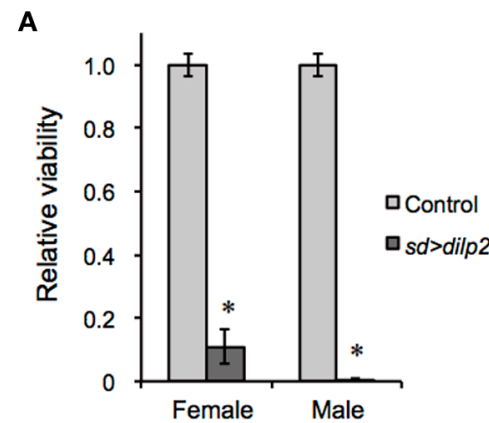

D

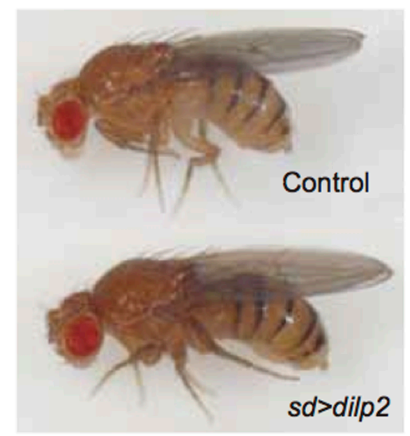

B

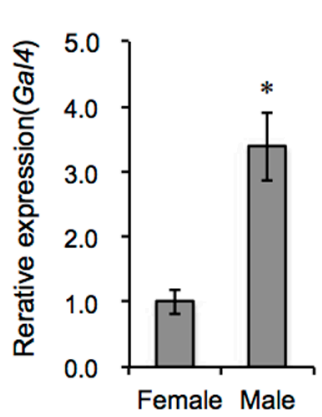

E

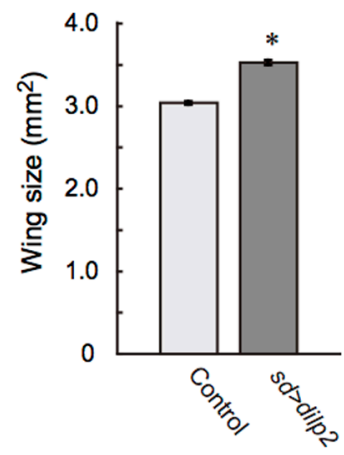

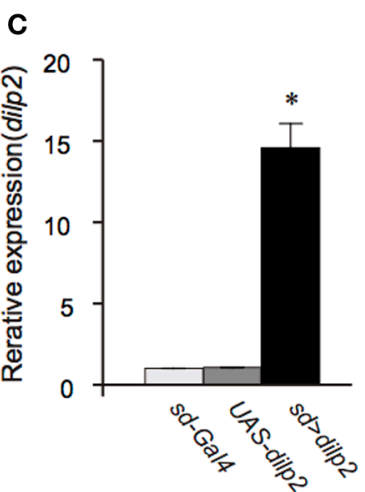

$\mathbf{F}$

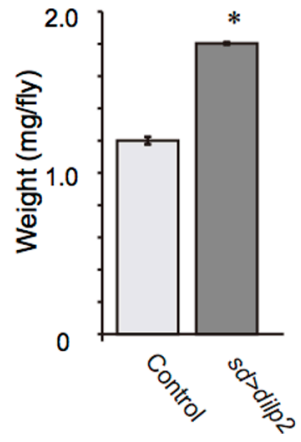

FIGURE 1 | Overexpression of dilp2 increases body size and causes semi-lethality. Relative viability of dilp2-overexpressing flies (A). Flies homozygous for $s d-G a / 4$ were crossed with UAS-dilp2/3xP3-RFP males, and the number of flies expressing RFP served as an internal control; those not expressing RFP are expressing dilp2. Expression level of Gal4 in sd-Gal4 male is much higher than that of $s d-G a l 4$ female (B). Overexpression of dilp2 caused semi-lethality for both males and females. The relative expression level of dilp2 mRNA in the dilp2-overexpressing flies ( $s d>$ dilp2) and the parental lines ( $s d-G a l 4$ and UAS-dilp2) served as controls and was determined by real-time RT-PCR (C). dilp2-overexpressing flies have increased body size (D), increased wing area by $17 \%$ (E), and increased weight by $50 \%$ (F). Student $t$-test was performed to analyze statistical significance. ${ }^{*} p<0.01$. 


\section{RESULTS AND DISCUSSION}

\section{OVEREXPRESSION OF dilp2 REDUCED THE EGG-TO-ADULT VIABILITY} OF THE FLIES

To generate a transgenic fly model of hyperinsulinemia, we overexpressed dilp2 which is known to promote tissue growth in Drosophila. We found that flies show high lethality when dilp2 was overexpressed ubiquitously using actin $5 c$-Gal4, suggesting

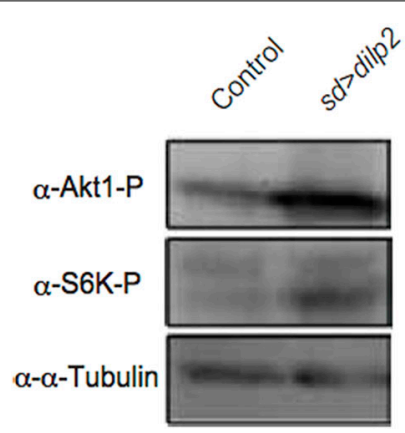

FIGURE 2 | dilp2-overexpression activates Akt1 and S6 kinase (S6K). The amount of phosphorylated Akt1 and S6K were analyzed by western blots. $\alpha$-tubulin was used as a loading control.
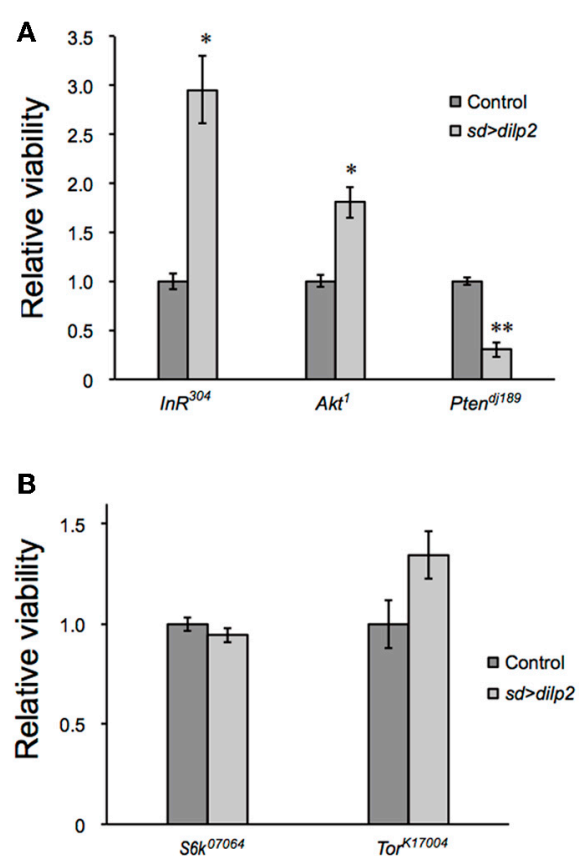

FIGURE 3 | Genetic manipulation of InR/Akt1/PI3K modifies the dilp2-induced lethality. Relative viability of the $s d>$ dilp2 flies was determined in combination with loss of function mutations in InR, Akt1, or PI3K, which are components of the insulin/IGF signaling pathway. dilp2induced semi-lethality was markedly improved by reduction of $\ln R$ or $A k t 1$, but enhanced by reduction of PTEN (A). Relative viability of the $s d>$ dilp2 flies in combination with loss-of-function mutations in Tor and S6K (B). The Tor/S6K pathway may not contribute to mediating the semi- lethality of dilp2-overexpressing flies. Student $t$-test was performed to analyze statistical significance. ${ }^{*} p<0.05,{ }^{* *} p<0.01$ that high levels of Dilp2 are toxic to flies. However, the reason for this lethality was not understood. An alternative driver, which gives a milder phenotype, would be useful to investigate the developmental toxicity associated with dilp2 overexpression. We tested several Gal4 lines, and found that $s d$-Gal4, which predominantly expresses Gal4 in developing imaginal wing discs, was an appropriate driver. When $s d$-Gal4 was crossed with UAS-dilp2,
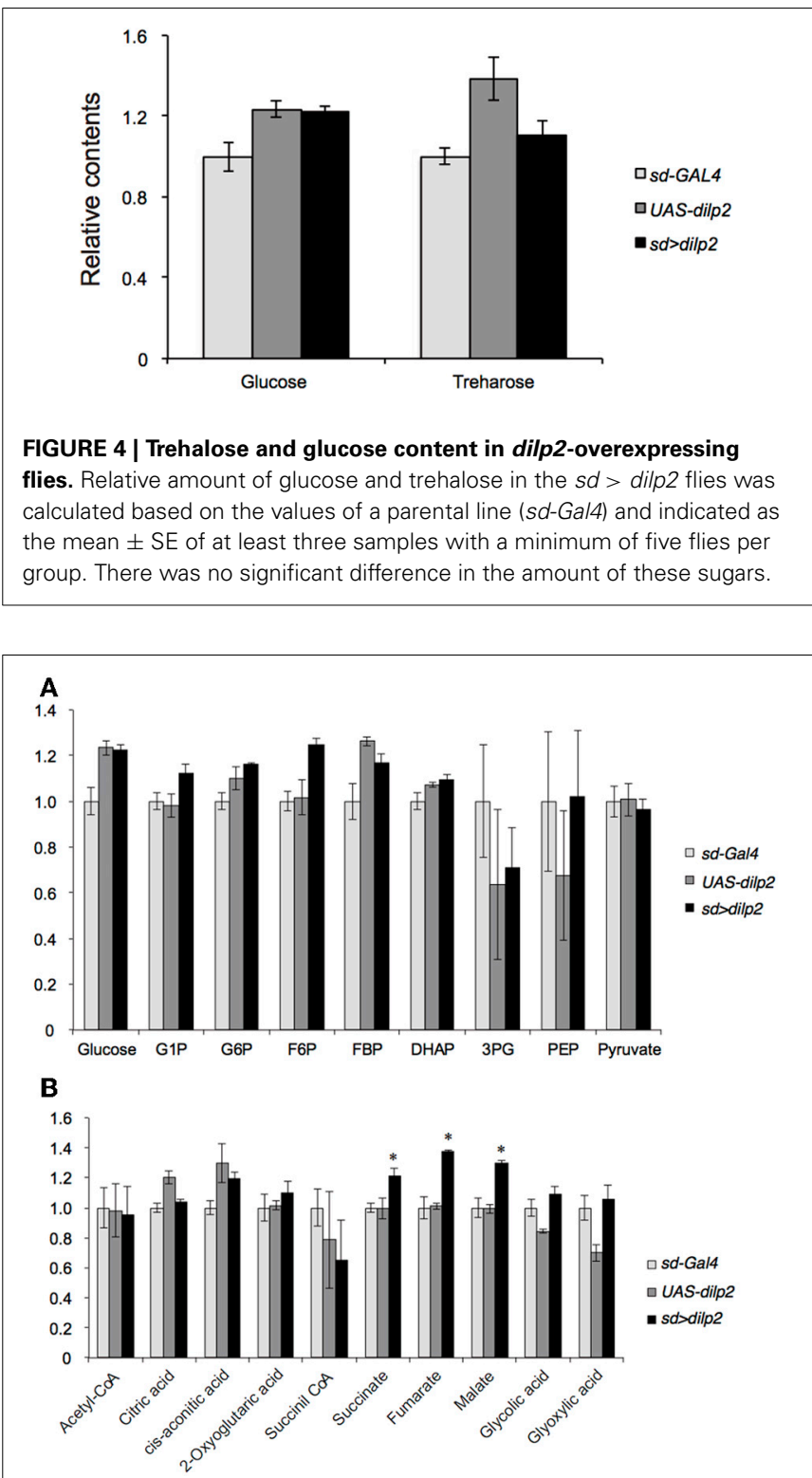

FIGURE 5 | Glucose metabolism in dilp2-overexpressing flies. Glycolysis (A) and TCA cycle (B) metabolites were analyzed by using LC-MS/MS in negative mode. Relative amounts of metabolites in the $s d>$ dilp2 flies were calculated based on the values of the parental lines (sd-Ga/4) and shown as the mean \pm S.E of at least three samples with a minimum of five flies per group. G6P: glucose 6-phosphate;F6P: fructose 6-phosphate; F1,6BP: fructose 1,6-bisphosphate; 2/3-PG: 2/3-phophoglycerate; PEP: phosphoenolpyruvate. Student $t$-test was performed to analyze statistical significance. ${ }^{*} p<0.05$ 


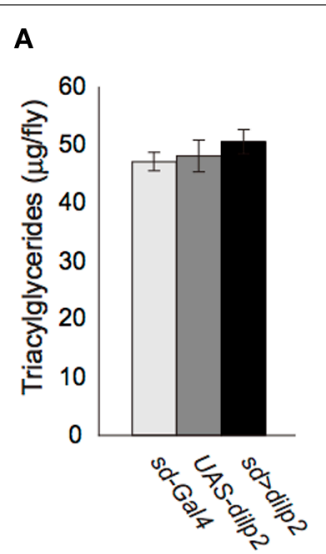

B

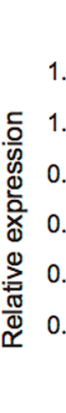

FIGURE 6 | Lipid metabolism in dilp2-overexpressing flies. Comparison of TAG levels between the dilp2-overexpressing flies and the parental lines as controls (A). Relative mRNA levels of four major genes involved in lipid catabolism in Drosophila, doppelganger von brummer (dob), brummer (bmm), CG5966, and CG11055 (B). Results are shown as the mean \pm SE of at least three experiments. ANOVA with Tukeys HSD was performed to analyze statistical significance. $* p<0.01$.

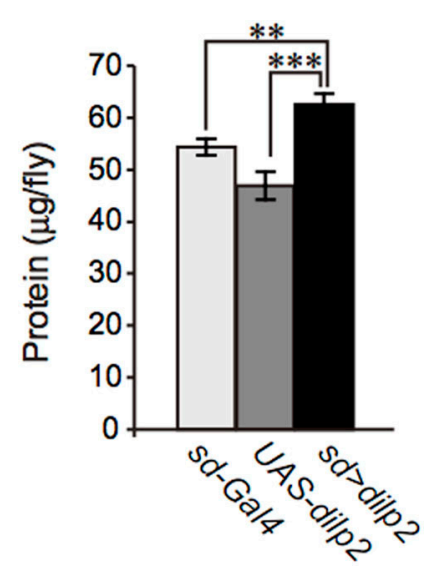

FIGURE 7 | Protein content in dilp2-overexpressing flies. The protein content was significantly increased in the dilp2-overexpressing flies ( $s d>$ dilp2) compared to the parental lines (sd-Gal4 and UAS-dilp2) as controls. Results are shown as the mean \pm SE of at least three experiments. Student $t$-test was performed to analyze statistical significance. ${ }^{* *} p<0.01$; *** $p<0.001$

the number of progenies from the cross was significantly less than the number of progenies obtained from the parental lines, thereby suggesting that overexpression of dilp2 was toxic to flies. To more quantitatively determine the viability, the number of progenies were compared between the dilp2-overexpressing flies and their siblings bearing the $M\{3 x P 3-R F P$.attP $\} Z H-68 E$ chromosome expressing red fluorescence protein (RFP) under the control of an artificial $3 x P 3$ promoter (Bischof et al., 2007). Female flies homozygous for sd-Gal4 were crossed to UAS-dilp2/M\{\{3xP3RFP.attP\}ZH-68E heterozygous males and the number of adult flies was counted. In theory, one half of the progeny inherit and express UAS-dilp2 under control of $s d$-Gal4, and the other half, serving as an internal control, carry an RFP-bearing chromosome. The number of dilp2-overexpressing flies was markedly

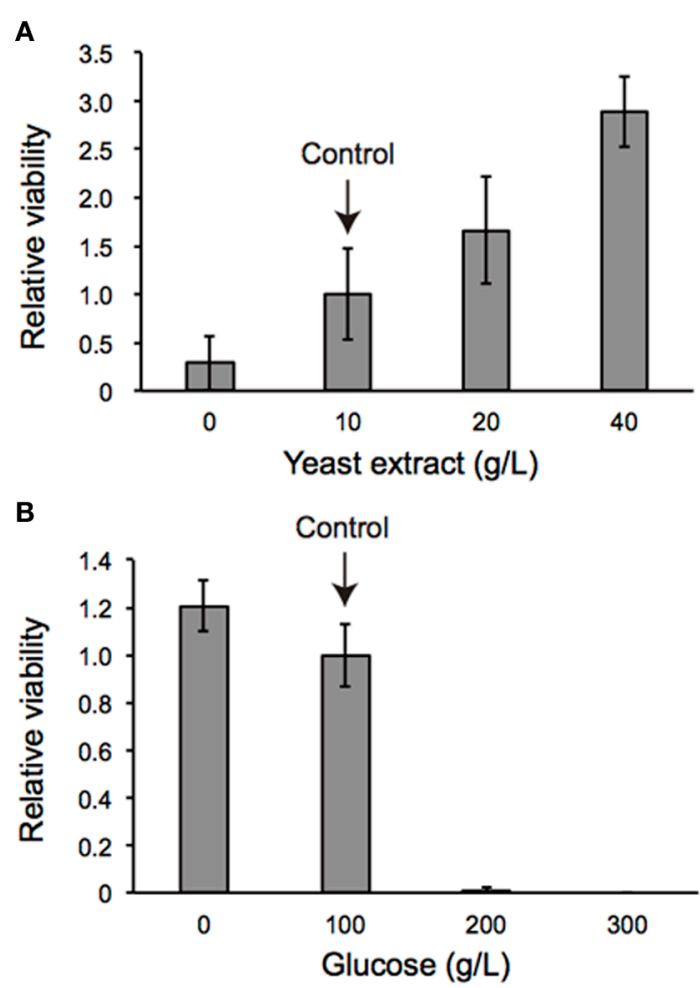

FIGURE 8 | Viability of dilp2-overexpressing

flies depends on nutritional conditions. Dilp2-overexpressing flies ( $s d>$ dilp2) were reared on media supplemented with various concentrations of yeast extract $(\mathbf{A})$ or glucose $\mathbf{( B )}$. Relative viability of the

dilp2-overexpressing flies ( $s d>$ dilp2) for each medium was calculated based on the number of control siblings.

reduced compared to that of the control flies (Figure 1A). Both sexes are semi-lethal, but the effects were more severe in the males than in the females. This is likely due to the dosage compensation mechanism, since $s d$-Gal4 is an X-linked transgene, 


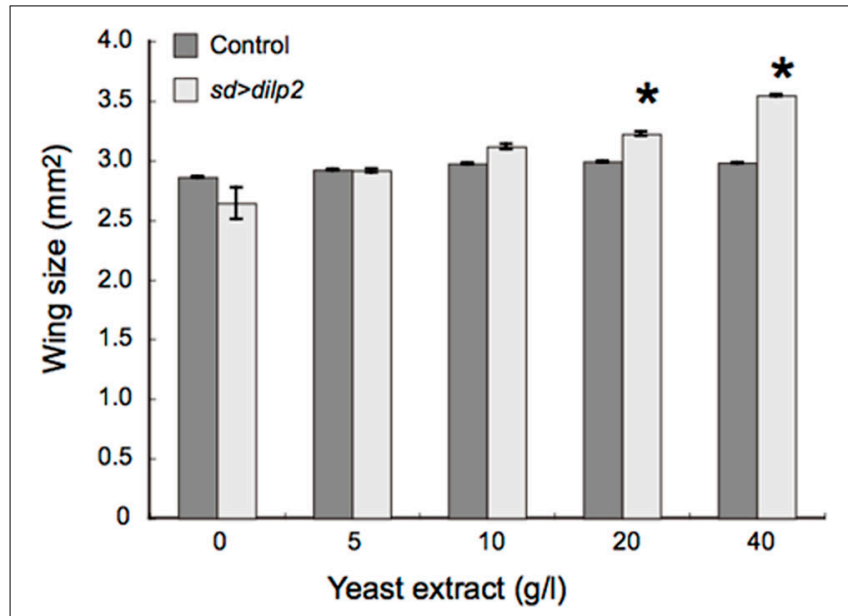

FIGURE 9 | Effects of protein-rich media on the wing size of dilp2-overexpressing flies. dilp2-overexpressing flies ( $s d>$ dilp2) were reared on media supplemented with various concentrations of yeast extract and their wing sizes were compared with those of the internal control flies. Mean wing area was positively correlated with the concentration of yeast extract in the media. Student $t$-test was performed to analyze statistical significance. ${ }^{*} p<0.05$

which drives expression of dilp2 two-times higher in males (Figure 1B).

Using female flies that survived to adult stage, we determined the expression level of dilp2 in females. Quantitative real-time PCR revealed that the levels of dilp 2 mRNA in the adult female increased 16-times higher than that of the control, suggesting that overexpression of dilp2 occurred and is toxic to fly development (Figure 1C).

We also noticed that the body size of the $s d>d i l p 2$ flies that survived to the adult stage was larger than that of the control (Figure 1D). To compare the body size more precisely, wing size was measured as described in the Materials and Methods section. The wing size of the $s d>$ dilp2 flies was increased by $17 \%$ compared to the wing size of the control flies (Figure 1E). In addition, the body weight of the dilp2-overexpressing flies increased by $50 \%$ compared to the control flies (Figure 1F). These results indicated that overexpression of dilp2 caused semi-lethality, but promoted growth for the survivors.

\section{THE PI3K/Akt1PATHWAY MEDIATES dilp2-INDUCED SEMI-LETHALITY}

dilp2 stimulates the PI3K/Akt1 pathway through insulin receptor (InR) activation. Western blotting analysis revealed that the active form of Akt1 was significantly increased in the dilp2overexpressing flies compared to the control, indicating that overexpression of dilp2 indeed activates the PI3K/Akt1 signaling (Figure 2 upper panel). To further confirm this, we investigated whether the mutations in the pathway components had an effect on dilp2-induced semi-lethality. We found that, for both males and females, the heterozygous flies that carry a lossof-function mutation in InR or Aktl showed higher levels of viability compared to the control flies (Figure 3A). On the other hand, a loss-of-function mutation in Ptne, a negative regulator of PI3K, further reduced the viability of dilp2-overexpressing

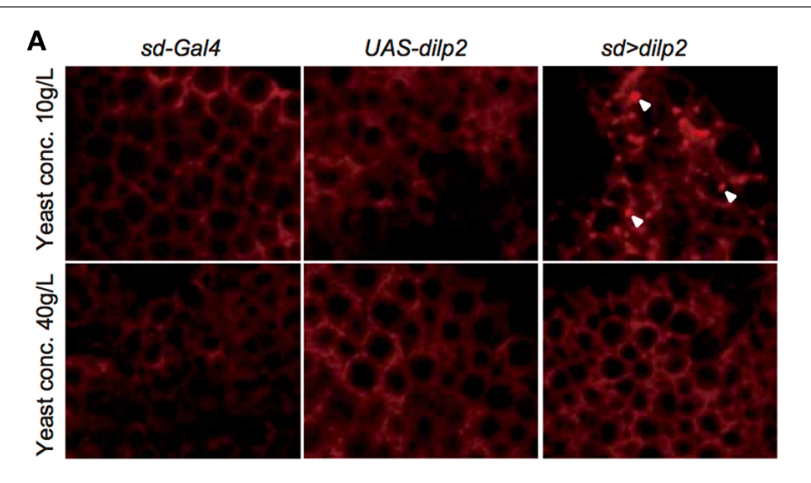

B

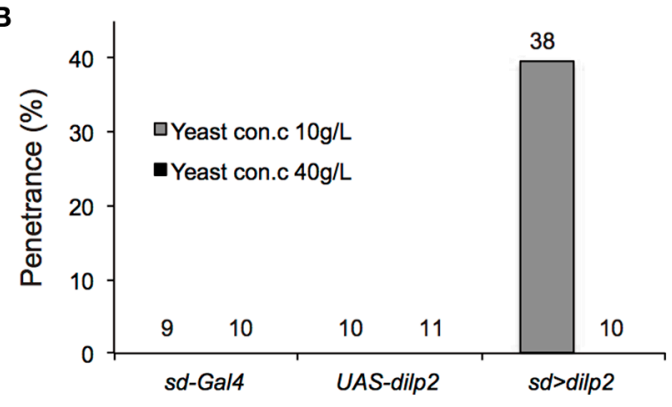

FIGURE 10 | Intensive autophagy in dilp2-overexpressing flies. Fat bodies were dissected from early third instar larvae overexpressing dilp2 ( $s d>$ dilp2) and stained with LysoTracker Red DND-99 (A). When the flies were reared on standard media containing $10 \mathrm{~g}$ yeast/L, strong punctate signals (arrowheads), corresponding to autophagy were observed in the $s d>$ dilp2 flies (upper right panel) but not in the parental lines ( $s d-G a l 4$ or UAS-dilp2) as the controls (upper left and middle panels). Autophagy was not detectable in the $s d>$ dilp2 flies when they were reared on protein-rich media containing $40 \mathrm{~g}$ yeast/L (lower panels). The penetrance (frequency) of ectopic autophagy in $s d>$ dilp2 flies (B). Numbers of samples observed were indicated above each bar.

flies (Figure 3A). These results suggest that dilp2-induced semilethality is mediated by the PI3K/Akt1 signaling. Overexpression of IlpP2 also activated the Tor/S6K signaling, a downstream signal component of the PI3K/Akt1 signaling, since the level of phosphorylated S6K was increased (Figure 2 middle panel). However, interestingly, the loss-of-function mutations in Tor or S6K had no effect on the reduced viability of the dilp2-overexpressing flies, suggesting that the Tor/S6K signaling is irrelevant to the semilethality or the single copies of mutations were not sufficient to suppress the dilp2-induced semi-lethality (Figure 3B).

\section{GLUCOSE AND LIPID METABOLISM IN dilp2 OVEREXPRESSING FLIES}

Next, we examined whether overexpression of dilp2 disrupts glucose homeostasis and metabolism. We first measured the concentration of glucose and trehalose, the major blood sugars in insects. There was no significant difference in the concentration of these sugars between the dilp2-overexpressing flies $(s d>d i l p 2)$ and the parental lines (sd-Gal4 or UAS-dilp2) as the controls (Figure 4). We also measured the amounts of glycolysis and TCA cycle metabolites using liquid chromatography coupled to tandem mass spectrometry (LC-MS/MS) with ionpair reagents (Figures 5A,B). There was no significant difference 


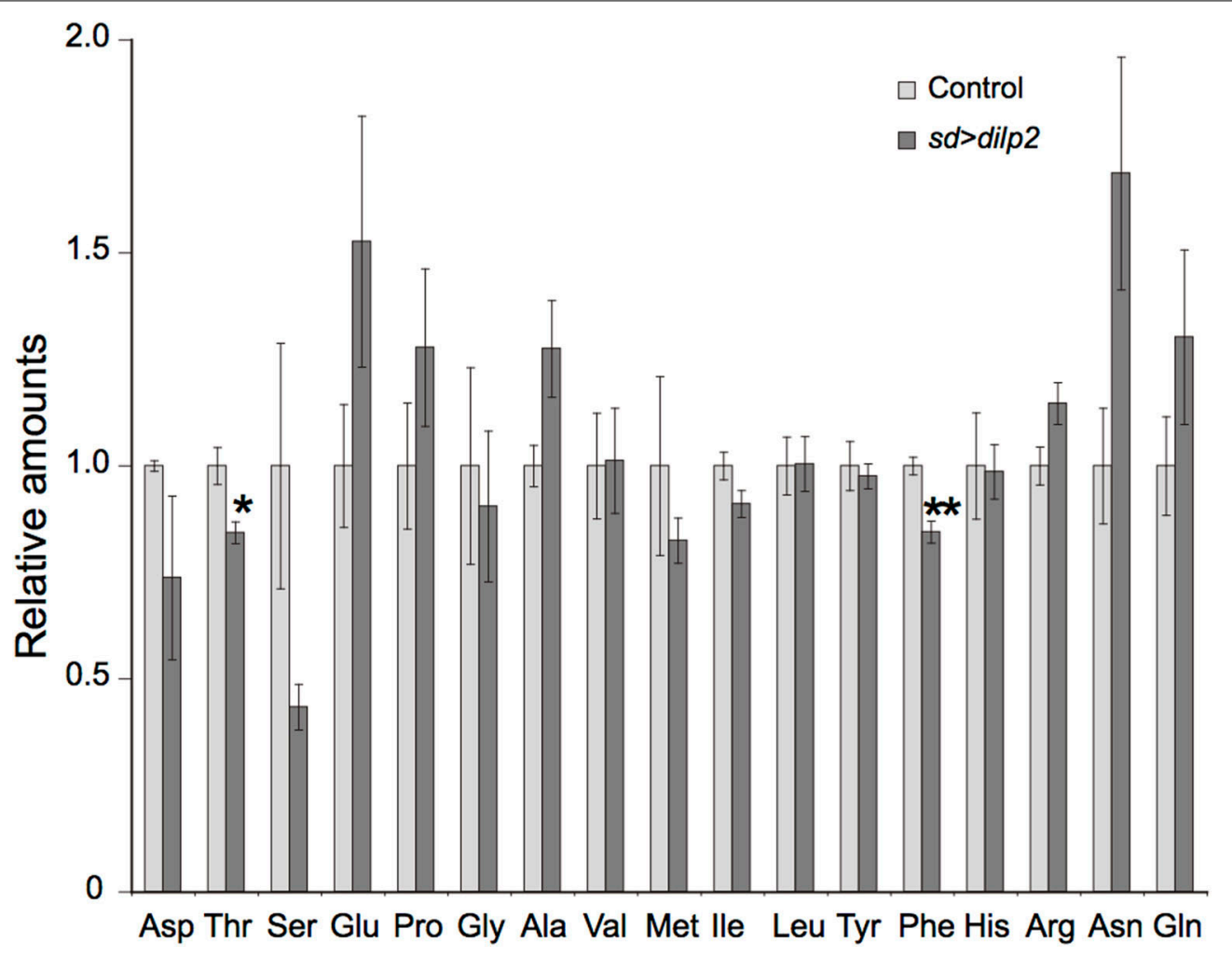

FIGURE 11 Amino acid content in dilp2-overexpressing flies. A free amino acids in the adult flies were quantified by using an LC-MS/MS in positive mode. Relative amounts of each amino acid in $s d>$ dilp2 flies were calculated based on those in the $s d>R F P$ flies as a control, and are shown as the mean \pm SE of at least three experiments with a minimum of five flies per group. Student $t$-test was performed to analyze statistical significance. ${ }^{*} p<0.05$, $* * p<0.01$. in the amounts of glycolytic metabolites between the dilp2overexpressing flies $(s d>d i l p 2)$ and the parental lines $(s d-G a l 4$ or UAS-dilp2) as the controls. These results suggest that glucose homeostasis itself was unaffected by overexpression of dilp2. For the TCA cycle metabolites, the amount of succinate, fumarate, and malate was slightly increased in the dilp2-overexpressing flies. Since fumarate and malate have been shown to extend lifespan in Caenorhabditis elegans (Edwards et al., 2013), overexpression of dilp2 might have affected the energy metabolism associated with lifespan determination.

Insulin/IGF signaling might down-regulate lipid catabolism via regulation of lipases (Xu et al., 2012). Thus, we next measured the lipid content in the dilp2-overexpressing flies and the control parental lines. There was no significant difference in the level of triacylglycerol, the most abundant of the storage lipids (Figure 6A). In addition, quantitative real-time PCR analysis revealed that the expression levels of four major Drosophila lipases, doppelganger von brummer (dob), brummer (bmm), CG5966, and CG11055 (Gronke et al., 2005) were not altered in the dilp2-overexpressing flies (Figure 6B). Expression level of $\mathrm{bmm}$ in the parental line, UAS-dilp2 was significantly lower than those of another parental line $s d$-Gal4 and of their progeny $s d>$ dilp2. There might be unknown mechanisms that downregulate $\mathrm{bmm}$ expression in the background of UAS-dilp2 line. We also compared the expression levels of $\mathrm{bmm}$ between $s d>d i l p 2$ and $s d-G a l 4,3 x P 3-R F P$ flies, and found that there was no significant change in $\mathrm{bmm}$ expression level when dilp2 was overexpressed (data not shown).

These results suggested that overexpression of dilp2 does not affect the lipid storage and catabolism in flies.

\section{NUTRIENT DEPENDENT EFFECTS OF dilp2-OVEREXPRESSION}

We also examined the protein content per fly and found that the dilp2-overexpressing flies contain more protein than the parental control lines (sd-Gal4 and UAS-dilp2), thereby suggesting that protein synthesis is elevated in $s d>d i l p 2$ flies (Figure 7). Interestingly, the viability of the dilp2-overexpressing flies increased depending on the concentration of the yeast extracts in the media (Figure 8A; Spearman's rank correlation coefficient $=0.79, p<0.0003)$. The relative viability of the dilp2overexpressing female flies was only $2 \%$ in the medium without yeast extract, whereas it was $16.6 \%$ in the medium containing $40 \mathrm{~g} / \mathrm{L}$ yeast extract. In addition, increasing protein content in the media significantly increased the mean wing area of the dilp2overexpressing flies, while it did not affect the wing size of the control flies (Figure 9). These results indicated that to support their development the dilp2-overexpressing flies require more protein as a nutrient than the control flies. It is possible that a shortage of protein sources occurs in the dilp2-overexpressing flies. To test this hypothesis, we examined whether autophagy 


\section{$s d>$ dilp2}

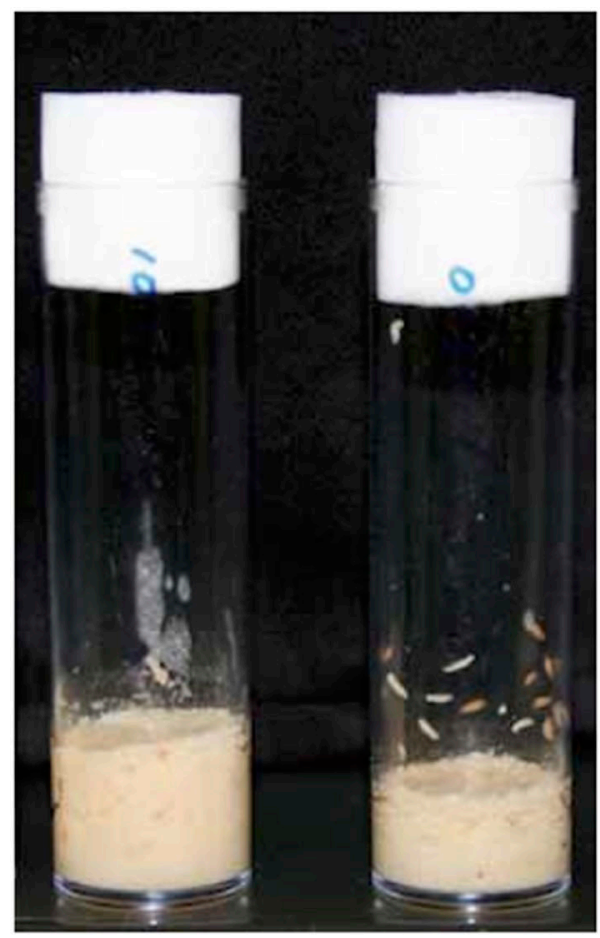

$100 \mathrm{~g} / \mathrm{L}$

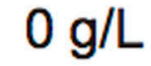

\section{Glucose contents}

FIGURE 12 | dilp2-overexpressing flies develop faster in media with reduced glucose. Overexpression of dilp2 retarded development of flies when they were reared on the standard media containing $100 \mathrm{~g} / \mathrm{L}$ glucose (left). However, flies develop faster when kept in glucose-deprived media (right). The mean ( \pm SE) durations of egg-to-early pupa was $106.3 \pm 1.3$ and $82.6 \pm 1.3 \mathrm{~h}$, with control media and glucose-deprived media, respectively (Student $t$-test: $p<0.001$ ).

occurs in the fat bodies dissected from the early third instar larvae. A large number of autophagy positive-cells were observed in the fat bodies of the dilp2-overexpressing flies While no autophagy positive-cell was found in the fat bodies of the control flies, (Figure 10). Increasing protein content in the media significantly suppressed the dilp2-mediated autophagy, suggesting that the dilp2-overexpressing flies were suffering from an insufficiency of protein sources. Namely, the shortage of protein sources could be one of the reasons for dilp2-induced semi-lethality.

Overexpression of dilp2 might cause chronic deficiency of amino acids. To examine this possibility, we quantified free amino acids in the $s d>d i l p 2$ and the $s d>$ RFP flies using an LC-MS/MS. Although the relative amount of threonine and phenylalanine were significantly different between the two groups, all changes were subtle and are unlikely to affect the viability (Figure 11). These results suggested that survivors of the dilp2-overexpressing flies were maintaining normal amino acid homeostasis probably though inducing ectopic autophagy. However, considering the semi-lethality of the animals, those failed to maintain amino acid homeostasis might have died earlier during development.

\section{A PROTEIN-TO-CARBOHYDRATE RATIO IS CRITICAL FOR THE SURVIVAL OF dilp2-OVEREXPRESSING FLIES}

The occurrence of autophagy in dilp2-overexpressing flies suggested that the animals were suffered from the shortage of amino acids. It has been demonstrated that a protein-to-carbohydrate ratio can affect the lifespan and the fecundity of flies (Lee et al., 2008). It is possible that overexpression of dilp2 affected the optimal ratio of these nutrients for the flies' development. Thus, we examined whether the glucose content of the fly food could modify the dilp2-induced semi-lethality. Increasing glucose concentration in the media significantly reduced the viability of the dilp2-overexpressing flies, indicating that overexpression of dilp2 enhances the susceptibility to glucose. On the other hand, decreasing glucose content in the media significantly improved the viability of the dilp2-overexpressing flies (Figure 8B Spearman's rank correlation coefficient $=-0.86, p<0.0001)$. Interestingly, the dilp2-overexpressing flies kept in a glucosereduced condition grew significantly faster than those reared on standard media (Figure 12). The mean $( \pm S E)$ durations of egg-to-early pupa was $106.3 \pm 1.3$ and $82.6 \pm 1.3 \mathrm{~h}$, with control media and glucose-deprived media, respectively (Student $t$-test: $p<0.001)$. These results strongly suggested that a highprotein low-carbohydrate diet is optimal for the viability of dilp2-overexpressing flies.

In this study, we demonstrated that overexpression of dilp2 severely decreases the egg-to-adult viability of flies and induced a high frequency of ectopic autophagy in fat bodies of early third instar larvae. As in mammalian cells, nutrient starvation induces autophagy through inhibition of Tor activity in Drosophila (Scott et al., 2004). However, the dilp2-overexpression-dependent autophagy does not seem to be regulated by the down-regulation of Tor, since S6K, a downstream target of Tor, was strongly activated (Figure 2). Activated S6K may execute autophagy, since expression of activated $\mathrm{S} 6 \mathrm{~K}$ increases starvation-induced autophagy in the absence of Tor in Drosophila (Scott et al., 2004). It is possible that a hyperactivation of S6K in the dilp2overexpressing flies might contribute to promoting autophagy. In isolated rat hepatocytes, some amino acids inhibit induction of autophagy in an mTor (mammalian Tor) independent manner (Kanazawa et al., 2004). Therefore, a shortage of protein sources may induce autophagy directly. The dilp2overexpression-dependent autophagy was reverted by a highprotein diet, suggesting that the nutritional condition is critical for survival of dilp2-overexpressing flies. It has been demonstrated that flies cultured on nutrient-rich food contain a significantly high level of secreted dilp2 compared to flies cultured on nutrient-deprived food, indicating that flies can sense the nutrient availability and modulate their insulin secretion accordingly (Geminard et al., 2009). Our results suggest that excess insulin/IGF signaling impairs the physiology of animals, which can be ameliorated by controlling the nutritional balance between proteins and carbohydrates, at least in flies. 


\section{ACKNOWLEDGMENTS}

We thank the Drosophila Genetic Resource Center, Kyoto, Japan and the Bloomington Stock Center for providing the fly stocks. This work was supported by a special grant from the Tokyo Metropolitan Government to Toshiro Aigaki.

\section{REFERENCES}

Bischof, J., Maeda, R. K., Hediger, M., Karch, F., and Basler, K. (2007). An optimized transgenesis system for Drosophila using germ-line-specific phiC31 integrases. Proc. Natl. Acad. Sci. U.S.A. 104, 3312-3317. doi: 10.1073/pnas.0611511104

Brogiolo, W., Stocker, H., Ikeya, T., Rintelen, F., Fernandez, R., and Hafen, E. (2001). An evolutionarily conserved function of the Drosophila insulin receptor and insulin-like peptides in growth control. Curr. Biol. 11, 213-221. doi: 10.1016/S0960-9822(01)00068-9

Broughton, S., Alic, N., Slack, C., Bass, T., Ikeya, T., Vinti, G., et al. (2008). Reduction of DILP2 in Drosophila triages a metabolic phenotype from lifespan revealing redundancy and compensation among DILPs. PLoS ONE 3:e3721. doi: 10.1371/journal.pone.0003721

Broughton, S. J., Piper, M. D., Ikeya, T., Bass, T. M., Jacobson, J., Driege, Y., et al. (2005). Longer lifespan, altered metabolism, and stress resistance in Drosophila from ablation of cells making insulin-like ligands. Proc. Natl. Acad. Sci. U.S.A. 102, 3105-3110. doi: 10.1073/pnas.0405775102

Corkey, B. E. (2012). Banting lecture 2011: hyperinsulinemia: cause or consequence? Diabetes 61, 4-13. doi: 10.2337/db11-1483

Edwards, C. B., Copes, N., Brito, A. G., Canfield, J., and Bradshaw, P. C. (2013). Malate and fumarate extend lifespan in Caenorhabditis elegans. PLoS ONE 8:e58345. doi: 10.1371/journal.pone.0058345

Gao, X., Neufeld, T. P., and Pan, D. (2000). Drosophila PTEN regulates cell growth and proliferation through PI3K-dependent and -independent pathways. Dev. Biol. 221, 404-418. doi: 10.1006/dbio.2000.9680

Geminard, C., Rulifson, E. J., and Leopold, P. (2009). Remote control of insulin secretion by fat cells in Drosophila. Cell Metab. 10, 199-207. doi: 10.1016/j.cmet.2009.08.002

Gronke, S., Mildner, A., Fellert, S., Tennagels, N., Petry, S., Muller, G., et al. (2005). Brummer lipase is an evolutionary conserved fat storage regulator in Drosophila. Cell Metab. 1, 323-330. doi: 10.1016/j.cmet.2005.04.003

Ikeya, T., Galic, M., Belawat, P., Nairz, K., and Hafen, E. (2002). Nutrientdependent expression of insulin-like peptides from neuroendocrine cells in the CNS contributes to growth regulation in Drosophila. Curr. Biol. 12, 1293-1300. doi: 10.1016/S0960-9822(02)01043-6

Kanazawa, T., Taneike, I., Akaishi, R., Yoshizawa, F., Furuya, N., Fujimura, S., et al. (2004). Amino acids and insulin control autophagic proteolysis through different signaling pathways in relation to mTOR in isolated rat hepatocytes. J. Biol. Chem. 279, 8452-8459. doi: 10.1074/jbc.M306337200

Lee, K. P., Simpson, S. J., Clissold, F. J., Brooks, R., Ballard, J. W., Taylor, P. W., et al. (2008). Lifespan and reproduction in Drosophila: new insights from nutritional geometry. Proc. Natl. Acad. Sci. U.S.A. 105, 2498-2503. doi: 10.1073/pnas.0710787105

Lushchak, O. V., Gospodaryov, D. V., Rovenko, B. M., Glovyak, A. D., Yurkevych, I. S., Klyuba, V. P., et al. (2012). Balance between macronutrients affects life span and functional senescence in fruit fly Drosophila melanogaster. J. Gerontol. A Biol. Sci. Med. Sci. 67, 118-125. doi: 10.1093/gerona/glr184

Montagne, J., Stewart, M. J., Stocker, H., Hafen, E., Kozma, S. C., and Thomas, G. (1999). Drosophila S6 kinase: a regulator of cell size. Science 285, 2126-2129. doi: $10.1126 /$ science.285.5436.2126
Musselman, L. P., Fink, J. L., Narzinski, K., Ramachandran, P. V., Hathiramani, S. S., Cagan, R. L., et al. (2011). A high-sugar diet produces obesity and insulin resistance in wild-type Drosophila. Dis. Model. Mech. 4, 842-849. doi: 10.1242/dmm.007948

Novosyadlyy, R., and LeRoith, D. (2010). Hyperinsulinemia and type 2 diabetes: impact on cancer. Cell Cycle 9, 1449-1450. doi: 10.4161/cc.9.8.11512

Oldham, S., Montagne, J., Radimerski, T., Thomas, G., and Hafen, E. (2000). Genetic and biochemical characterization of dTOR, the Drosophila homolog of the target of rapamycin. Genes Dev. 14, 2689-2694. doi: 10.1101/gad.845700

Rulifson, E. J., Kim, S. K., and Nusse, R. (2002). Ablation of insulin-producing neurons in flies: growth and diabetic phenotypes. Science 296, 1118-1120. doi: 10.1126/science. 1070058

Saltiel, A. R., and Kahn, C. R. (2001). Insulin signalling and the regulation of glucose and lipid metabolism. Nature 414, 799-806. doi: 10.1038/414799a

Samuel, V. T., and Shulman, G. I. (2012). Mechanisms for insulin resistance: common threads and missing links. Cell 148, 852-871. doi: 10.1016/j.cell.2012.02.017

Scott, R. C., Schuldiner, O., and Neufeld, T. P. (2004). Role and regulation of starvation-induced autophagy in the Drosophila fat body. Dev. Cell 7, 167-178. doi: 10.1016/j.devcel.2004.07.009

Skorokhod, A., Gamulin, V., Gundacker, D., Kavsan, V., Muller, I. M., and Muller, W. E. (1999). Origin of insulin receptor-like tyrosine kinases in marine sponges. Biol. Bull. 197, 198-206. doi: 10.2307/1542615

Skorupa, D. A., Dervisefendic, A., Zwiener, J., and Pletcher, S. D. (2008). Dietary composition specifies consumption, obesity, and lifespan in Drosophila melanogaster. Aging Cell 7, 478-490. doi: 10.1111/j.1474-9726.2008. 00400.x

Stocker, H., Andjelkovic, M., Oldham, S., Laffargue, M., Wymann, M. P., Hemmings, B. A., et al. (2002). Living with lethal PIP3 levels: viability of flies lacking PTEN restored by a PH domain mutation in Akt/PKB. Science 295, 2088-2091. doi: 10.1126/science.1068094

Tsuda, M., Kobayashi, T., Matsuo, T., and Aigaki, T. (2010). Insulindegrading enzyme antagonizes insulin-dependent tissue growth and Abetainduced neurotoxicity in Drosophila. FEBS Lett. 584, 2916-2920. doi: 10.1016/j.febslet.2010.05.010

Xu, X., Gopalacharyulu, P., Seppanen-Laakso, T., Ruskeepaa, A. L., Aye, C. C., Carson, B. P., et al. (2012). Insulin signaling regulates fatty acid catabolism at the level of CoA activation. PLoS Genet. 8:e1002478. doi: 10.1371/journal.pgen. 1002478

Conflict of Interest Statement: The authors declare that the research was conducted in the absence of any commercial or financial relationships that could be construed as a potential conflict of interest.

Received: 08 February 2014; accepted: 28 March 2014; published online: 16 April 2014. Citation: Sato-Miyata Y, Muramatsu K, Funakoshi M, Tsuda M and Aigaki T (2014) Overexpression of dilp2 causes nutrient-dependent semi-lethality in Drosophila. Front. Physiol. 5:147. doi: 10.3389/fphys.2014.00147

This article was submitted to Invertebrate Physiology, a section of the journal Frontiers in Physiology.

Copyright (c) 2014 Sato-Miyata, Muramatsu, Funakoshi, Tsuda and Aigaki. This is an open-access article distributed under the terms of the Creative Commons Attribution License (CC BY). The use, distribution or reproduction in other forums is permitted, provided the original author (s) or licensor are credited and that the original publication in this journal is cited, in accordance with accepted academic practice. No use, distribution or reproduction is permitted which does not comply with these terms. 